MATHEMATICS OF COMPUTATION

Volume 70, Number 234, Pages 789-799

S 0025-5718(00)01211-4

Article electronically published on March 1, 2000

\title{
A CLASS OF INFINITE SUMS AND INTEGRALS
}

\author{
R. SHAIL
}

\begin{abstract}
In this paper closed-form sums are given for various slowly-convergent infinite series which arise essentially from the differentiation of Dirichlet $L$-series. Some associated integrations are also considered. A small number of the results appear in standard tables, but most seem to be new.
\end{abstract}

\section{INTRODUCTION}

The Dirichlet $L$-functions have long played an important role in various areas of analytic number theory, but recent research [1] has shown that they occur in the evaluation of lattice sums and lattice limits which arise in the mathematical study of crystal lattices and the Wigner model of a metal. Thus, two typical results of electrostatic energy calculations for lattices are [1]

$$
\begin{aligned}
\lim _{N \rightarrow \infty}\left(\sum_{m=-N}^{m=N} \sum_{n=-N}^{n=N}\left(m^{2}+n^{2}\right)^{-1 / 2}\right. & \left.-\int_{-N-1 / 2}^{N+1 / 2} \int_{-N-1 / 2}^{N+1 / 2}\left(x^{2}+y^{2}\right)^{-1 / 2} d x d y\right) \\
& =4 \zeta(1 / 2) L_{-4}(1 / 2),
\end{aligned}
$$

where $\zeta$ is the Riemann zeta function and $L_{-4}(1 / 2)$ a Dirichlet $L$-function, and [2]

$$
\begin{gathered}
\lim _{N \rightarrow \infty}\left(-\sum_{m=-N}^{m=N} \sum_{n=-N}^{n=N} \ln \left(m^{2}+n^{2}\right)\right. \\
\left.+\int_{-N-1 / 2}^{N+1 / 2} \int_{-N-1 / 2}^{N+1 / 2} \ln \left(x^{2}+y^{2}\right) d x d y\right) \\
=\ln \left(\frac{2}{\pi}\right)-2 \ln \left(\frac{\Gamma(1 / 4)}{\Gamma(3 / 4)}\right)+\frac{\pi}{6} .
\end{gathered}
$$

In (1.1) the $L$-function $L_{-4}(s)$ is defined for $\operatorname{Re} s>0$ as

$$
L_{-4}(s)=\sum_{n=0}^{\infty}(-1)^{n}(2 n+1)^{-s} .
$$

The terms with $m=n=0$ in (1.1) and (1.2) are omitted, and separately each of the double sums and integrals diverges as $N \rightarrow \infty$.

In the course of these investigations it became necessary to evaluate derivatives such as $L_{-4}^{\prime}(0)$, and this led to closed forms for the summations and integrations which are the subject of this note. These sums and integrals, of which a typical

Received by the editor May 5, 1999.

2000 Mathematics Subject Classification. Primary 65B10.

(C)2000 American Mathematical Society 
example closely related to (1.2) is

$$
\sum_{n=1}^{\infty}(-1)^{n+1} \frac{\ln (2 n+1)}{2 n+1}=\frac{\pi}{4}\left\{\gamma+\ln (2 \pi)-2 \ln \left(\frac{\Gamma(1 / 4)}{\Gamma(3 / 4)}\right)\right\}
$$

do not appear in the standard tables [3], and hence it seems worthwhile to record them and the simple steps which produce them.

\section{Characters And Dirichlet $L$-FunCtions}

Let $k$ be a positive integer and $\chi_{k}(n)$ a real number-theoretic character modulo $k$ with the defining properties [4]

$$
\begin{gathered}
\chi_{k}(1)=1, \chi_{k}(n)=\chi_{k}(n+k), \\
\chi_{k}(m) \chi_{k}(n)=\chi_{k}(m n) \text { for all } m, n, \\
\chi_{k}(n)=0 \text { if }(k, n) \neq 1 .
\end{gathered}
$$

Thus nonzero characters take only the values \pm 1 and, apart from the principal character (in which all nonzero values are unity), there are equal numbers of both values, i.e.

$$
\sum_{n=1}^{k} \chi_{k}(n)=0
$$

The Dirichlet $L$-functions $L_{ \pm k}(s)$, where $s$ is a complex variable, are defined by

$$
L_{ \pm k}(s)=\sum_{n=1}^{\infty} \chi_{k}(n) n^{-s}
$$

wherein the subscript $\pm k$ is used according as $\chi_{k}(k-1)= \pm 1$. For nonprincipal characters $\chi_{k}(n)$ all the series for $L_{ \pm k}(s)$ converge for Re $s>0$ 4. However, when $\chi_{k}(n)$ is a principal character, $L_{ \pm k}(s)$ has a simple pole at $s=1$; for example, when $k=1, L_{1}(s)$ is the Riemann zeta function $\zeta(s)$ and (2.2) converges for $\operatorname{Re} s>1$. In this paper all characters are required to be nonprincipal.

The Dirichlet functions satisfy the functional equations [5]

$$
\begin{aligned}
& L_{-k}(s)=2^{s} \pi^{s-1} k^{-s+\frac{1}{2}} \cos (s \pi / 2) L_{-k}(1-s) \Gamma(1-s), \\
& L_{+k}(s)=2^{s} \pi^{s-1} k^{-s+\frac{1}{2}} \sin (s \pi / 2) L_{+k}(1-s) \Gamma(1-s),
\end{aligned}
$$

which can be used for analytic continuation into Re $s \leq 0$. A further useful integral representation [6] of the Dirichlet function states that for Re $s>0$,

$$
L_{ \pm k}(s) \Gamma(s)=\int_{0}^{\infty} \frac{u^{s-1}}{1-e^{-k u}}\left(\sum_{n=1}^{k} \chi_{k}(n) e^{-n u}\right) d u
$$

The Dirichlet $L$-functions are divided into two classes, primitive and nonprimitive. A nonprimitive $L$-function can be expressed in terms of simpler $L$-functions (for example, $\left.L_{-6}(s)=\left(1+2^{-s}\right) L_{-3}(s)\right)$, and here we restrict attention to primitive $L$-functions. A list of such functions, complete up to $L_{ \pm 8}(s)$, is given in [7], and further information appears in [6]. 
It is appropriate to define here the generalised zeta function $\zeta(s, a)$ for $\operatorname{Re} s>1$ by

$$
\zeta(s, a)=\sum_{n=0}^{\infty}(a+n)^{-s} .
$$

An alternative representation, due to Hermite and valid for all $s$, is [8]

$$
\zeta(s, a)=\frac{a^{-s}}{2}+\frac{a^{1-s}}{s-1}+2 \int_{0}^{\infty}\left(a^{2}+y^{2}\right)^{-s / 2} \sin (s \arctan (y / a)) \frac{d y}{e^{2 \pi y}-1},
$$

a form which exhibits $\zeta(s, a)$ as an analytic function of $s$ whose only singularity is a simple pole at $s=1$ with residue $a^{1-s}$. Using (2.6), the Dirichlet function $L_{ \pm k}(s)$ can be expressed, for $\operatorname{Re} s>1$, as

$$
L_{ \pm k}(s)=\sum_{n=1}^{\infty} \frac{\chi_{k}(n)}{n^{s}}=\frac{1}{k^{s}} \sum_{p=1}^{k} \chi_{k}(p) \zeta(s, p / k)
$$

and the second summation, together with (2.7), can be used to analytically continue $L_{ \pm k}(s)$ into $\operatorname{Re} s \leq 1$.

\section{Sums And Integrals When $\chi_{k}(k-1)=-1$}

The main results of this section are contained in the following theorem:

Theorem 3.1. Let $\chi_{k}(n)$ be a nonprincipal character with $\chi_{k}(k-1)=-1$. Then

$$
\sum_{n=2}^{\infty} \frac{\chi_{k}(n) \ln n}{n}=\frac{\pi}{k^{\frac{1}{2}}}\left\{\ln \prod_{p=1}^{k} \Gamma(p / k)^{\chi_{k}(p)}+\frac{1}{k}(\gamma+\ln (2 \pi)) \sum_{p=1}^{k} p \chi_{k}(p)\right\}
$$

and

$$
\begin{aligned}
& \int_{0}^{\infty} \frac{\ln u}{1-e^{-k u}}\left(\sum_{n=1}^{k} \chi_{k}(n) e^{-n u}\right) d u \\
& \quad=-\frac{\pi}{k^{\frac{1}{2}}}\left\{\ln \prod_{p=1}^{k} \Gamma(p / k)^{\chi_{k}(p)}+\frac{1}{k} \ln (2 \pi) \sum_{p=1}^{k} p \chi_{k}(p)\right\} .
\end{aligned}
$$

Proof. To establish the summation formula we first note that from (2.8) for $\operatorname{Re} s>0$ [4]

$$
L_{-k}^{\prime}(s)=-\sum_{n=2}^{\infty} \frac{\chi_{k}(n) \ln n}{n^{s}}
$$

Further, using (2.8) and appropriate analytic continuations of the generalised zeta functions, we also have that for all $s$

$$
L_{-k}^{\prime}(s)=\frac{1}{k^{s}} \sum_{p=1}^{k} \chi_{k}(p)\left\{-\ln k \zeta(s, p / k)+\zeta^{\prime}(s, p / k)\right\} .
$$

(When $s=1$ the right member of (3.2) is interpreted as the limit as $s \rightarrow 1$ of the finite sum over $p$; a similar remark applies to (2.8).) Thus, equations (2.8) and (3.2) define $L_{-k}(s)$ and $L_{-k}^{\prime}(s)$ for all $s$. In particular, setting $s=0$, using the results (ㅍ. [1]

$$
\zeta(0, a)=\frac{1}{2}-a, \zeta^{\prime}(0, a)=\ln \Gamma(a)-\frac{1}{2} \ln (2 \pi)
$$


and the zero-sum property (2.1) of the character, we deduce from (2.8) and (3.2) that

$$
\begin{gathered}
L_{-k}(0)=-\frac{1}{k} \sum_{p=1}^{k} p \chi_{k}(p), \\
L_{-k}^{\prime}(0)=\sum_{p=1}^{k} \chi_{k}(p)\left\{\frac{p}{k} \ln k+\ln \Gamma(p / k)\right\} .
\end{gathered}
$$

We next use the functional equation $(2.3)$ to relate $L_{-k}^{\prime}(0)$ and $L_{-k}^{\prime}(1)$; differentiating (2.3) with respect to $s$ and setting $s=0$ gives

$$
L_{-k}^{\prime}(0)=\frac{k^{\frac{1}{2}}}{\pi}\left\{\ln \left(\frac{2 \pi}{k}\right) L_{-k}(1)-L_{-k}^{\prime}(1)-\Gamma^{\prime}(1) L_{-k}(1)\right\} .
$$

Further, letting $s \rightarrow 1$ in 2.3 ,

$$
L_{-k}(1)=\frac{\pi}{k^{\frac{1}{2}}} L_{-k}(0) .
$$

Thus, using the infinite-series expression for $L_{-k}^{\prime}(1)$ from (3.1), together with (3.4) through (3.7) and the value $\Gamma^{\prime}(1)=-\gamma$, we obtain after some simplification the sum formula for a character $\chi_{k}(n)$ with $\chi_{k}(k-1)=-1$,

$$
\sum_{n=2}^{\infty} \frac{\chi_{k}(n) \ln n}{n}=\frac{\pi}{k^{\frac{1}{2}}}\left\{\ln \prod_{p=1}^{k} \Gamma(p / k)^{\chi_{k}(p)}+\frac{1}{k}(\gamma+\ln (2 \pi)) \sum_{p=1}^{k} p \chi_{k}(p)\right\}
$$

To derive the integral in Theorem 3.1 we first differentiate the representation (2.5) with respect to $s$ and set $s=1$ to give

$$
L_{-k}^{\prime}(1)-\gamma L_{-k}(1)=\int_{0}^{\infty} \frac{\ln u}{1-e^{-k u}}\left(\sum_{n=1}^{k} \chi_{k}(n) e^{-n u}\right) d u .
$$

Using (3.1) with $s=1$, 3.4, (3.7) and (3.8) in (3.9), it now follows after some simple manipulation that

$$
\begin{aligned}
& \int_{0}^{\infty} \frac{\ln u}{1-e^{-k u}}\left(\sum_{n=1}^{k} \chi_{k}(n) e^{-n u}\right) d u \\
& \quad=-\frac{\pi}{k^{\frac{1}{2}}}\left\{\ln \prod_{p=1}^{k} \Gamma(p / k)^{\chi_{k}(p)}+\frac{1}{k} \ln (2 \pi) \sum_{p=1}^{k} p \chi_{k}(p)\right\}
\end{aligned}
$$

the required integration formula. For constant $\alpha>0$ a generalisation of (3.10) is

$$
\begin{aligned}
\int_{0}^{\infty} \frac{\ln u}{1-e^{-k \alpha u}} & \left(\sum_{n=1}^{k} \chi_{k}(n) e^{-n \alpha u}\right) d u \\
& =-\frac{\pi}{\alpha k^{\frac{1}{2}}}\left\{\ln \prod_{p=1}^{k} \Gamma(p / k)^{\chi_{k}(p)}+\frac{1}{k} \ln \left(\frac{2 \pi}{\alpha}\right) \sum_{p=1}^{k} p \chi_{k}(p)\right\},
\end{aligned}
$$

a formula which follows from (3.10) on replacing $u$ in the left member of (3.11) by $v / \alpha$, writing $\ln (v / \alpha)$ as a difference of logarithms and using (2.5) with $s=1$ to evaluate the integral multiplying $\ln \alpha$. 
We now give some examples of our formulae for selected values of $k$.

(a) $k=3$. The nonprincipal real character has nonzero values given by $\chi_{3}(1)=1$, $\chi_{3}(2)=-1$, and (3.8) becomes, after some slight rearrangement,

$$
\begin{aligned}
\sum_{n=0}^{\infty}\left\{\frac{\ln (3 n+1)}{3 n+1}-\frac{\ln (3 n+2)}{3 n+2}\right\} & =\frac{\pi}{3^{\frac{1}{2}}}\left\{\ln \left(\frac{\Gamma(1 / 3)}{\Gamma(2 / 3)}\right)-\frac{1}{3}(\gamma+\ln (2 \pi))\right\} \\
& =-0.2226629869680150949
\end{aligned}
$$

The integral (3.10) provides the result that

$$
\begin{aligned}
\int_{0}^{\infty} \frac{\ln u}{1+2 \cosh u} d u & =\frac{\pi}{3^{\frac{1}{2}}}\left\{\frac{1}{3} \ln (2 \pi)-\ln \left(\frac{\Gamma(1 / 3)}{\Gamma(2 / 3)}\right)\right\} \\
& =-0.12632148170620903637
\end{aligned}
$$

which is the special case of entry 4.371, 2 in 3 ] when $t=\pi / 3$.

(b) $k=4$. The nonzero values of the real nonprincipal character with $k=4$ are $\chi_{4}(1)=1$ and $\chi_{4}(3)=-1$; then Theorem 3.1 gives the results

$$
\begin{aligned}
\sum_{n=1}^{\infty}(-1)^{n+1} \frac{\ln (2 n+1)}{2 n+1} & =\frac{\pi}{4}\left\{\gamma+\ln (2 \pi)-2 \ln \left(\frac{\Gamma(1 / 4)}{\Gamma(3 / 4)}\right)\right\} \\
& =0.19290131679691242936
\end{aligned}
$$

and

$$
\begin{aligned}
\int_{0}^{\infty} \frac{\ln u}{\cosh u} d u & =\frac{\pi}{2}\left\{\ln (2 \pi)-2 \ln \left(\frac{\Gamma(1 / 4)}{\Gamma(3 / 4)}\right)\right\} \\
& =-0.52088561260197689108
\end{aligned}
$$

The summation (3.14) appears in 2], and the integral (3.15) in 3], entry 4.371, 1.

(c) $k=8$. There are two nonprincipal characters for $k=8$, and that with $\chi_{8}(7)=-1$ has the remaining nonzero values $\chi_{8}(1)=\chi_{8}(3)=1$ and $\chi_{8}(5)=-1$. The results supplied by Theorem 3.1 are now

$$
\begin{aligned}
\sum_{n=0}^{\infty}(-1)^{n}\left\{\frac{\ln (4 n+1)}{4 n+1}+\frac{\ln (4 n+3)}{4 n+3}\right\} & =\frac{\pi}{2 \sqrt{2}}\left\{\ln \left(\frac{\Gamma(1 / 8) \Gamma(3 / 8)}{\Gamma(5 / 8) \Gamma(7 / 8)}\right)-\gamma-\ln (2 \pi)\right\} \\
& =0.02300458786273601032
\end{aligned}
$$

and

$$
\begin{aligned}
\int_{0}^{\infty} \ln u \frac{\cosh u}{\cosh 2 u} d u & =\frac{\pi}{2 \sqrt{2}}\left\{-\ln \left(\frac{\Gamma(1 / 8) \Gamma(3 / 8)}{\Gamma(5 / 8) \Gamma(7 / 8)}\right)+\ln (2 \pi)\right\} \\
& =-0.66412999516992532907 .
\end{aligned}
$$

It is of interest to note that [3] gives the integral (3.17) with lower limit 1 (entry 4.372, 2 with $m=1, n=2$ ) but not (3.17) itself.

(d) $k=20$. For $k=20$ there is a real character with nonzero values $\chi_{20}(1)=$ $\chi_{20}(3)=\chi_{20}(7)=\chi_{20}(9)=1$ and $\chi_{20}(11)=\chi_{20}(13)=\chi_{20}(17)=\chi_{20}(19)=-1$. 
The summation (3.8) reads

$$
\begin{aligned}
\sum_{n=0}^{\infty}(-1)^{n}\left\{\frac{\ln (10 n+1)}{10 n+1}\right. & \left.+\frac{\ln (10 n+3)}{10 n+3}+\frac{\ln (10 n+7)}{10 n+7}+\frac{\ln (10 n+9)}{10 n+9}\right\} \\
& =\frac{\pi}{2 \sqrt{5}}\left\{\ln \left(\frac{\boldsymbol{\Gamma}(1,3,7,9 ; 20)}{\boldsymbol{\Gamma}(11,13,17,19 ; 20)}\right)-2(\gamma+\ln (2 \pi))\right\} \\
& =0.44609603117958983760,
\end{aligned}
$$

where

$$
\Gamma\left(a_{1}, a_{2}, \ldots, a_{m} ; k\right)=\Gamma\left(a_{1} / k\right) \Gamma\left(a_{2} / k\right) \cdots \Gamma\left(a_{m} / k\right) .
$$

The integral of Theorem 3.1 reduces to

$$
\begin{aligned}
& \int_{0}^{\infty} \ln u \frac{\cosh 3 u}{2 \cosh 4 u-2 \cosh 2 u+1} d u \\
& \quad= \frac{\pi}{4 \sqrt{5}}\left\{-\ln \left(\frac{\boldsymbol{\Gamma}(1,3,7,9 ; 20)}{\boldsymbol{\Gamma}(11,13,17,19 ; 20)}\right)+2 \ln (2 \pi)\right\} \\
& \quad=-0.62853132616857048038 .
\end{aligned}
$$

Rather than give further examples of Theorem 3.1 we prefer to indicate a generalisation which arises by differentiating (3.1) with respect to $s$ and setting $s=1$ to obtain

$$
L_{-k}^{\prime \prime}(1)=\sum_{n=2}^{\infty} \frac{\chi_{k}(n)(\ln n)^{2}}{n}
$$

It also follows from (2.8) that

$$
L_{-k}^{\prime \prime}(s)=\frac{1}{k^{s}} \sum_{p=1}^{k} \chi_{k}(p)\left\{(\ln k)^{2} \zeta(s, p / k)-2 \ln k \zeta^{\prime}(s, p / k)+\zeta^{\prime \prime}(s, p / k)\right\} .
$$

The arguments leading to (3.8) can now be repeated mutatis mutandis to supply the summation

$$
\begin{array}{r}
\sum_{n=2}^{\infty} \frac{\chi_{k}(n)(\ln n)^{2}}{n}=\frac{\pi}{k^{\frac{1}{2}}}\left\{\sum_{p=1}^{k} \chi_{k}(p) \zeta^{\prime \prime}(0, p / k)-2(\ln (2 \pi)+\gamma) \ln \prod_{p=1}^{k} \Gamma(p / k)^{\chi_{k}(p)}\right. \\
\left.-\frac{1}{k}\left((\ln (2 \pi)+\gamma)^{2}+\frac{\pi^{2}}{12}\right) \sum_{p=1}^{k} p \chi_{k}(p)\right\},
\end{array}
$$

and the associated integral is found as

$$
\begin{aligned}
\int_{0}^{\infty} \frac{(\ln u)^{2}}{1-e^{-k u}}\left(\sum_{n=1}^{k} \chi_{k}(n) e^{-n u}\right) d u & \\
=\frac{\pi}{k^{\frac{1}{2}}}\left\{\sum_{p=1}^{k} \chi_{k}(p) \zeta^{\prime \prime}(0, p / k)\right. & -2 \ln (2 \pi) \ln \prod_{p=1}^{k} \Gamma(p / k)^{\chi_{k}(p)} \\
& \left.-\frac{1}{k}\left((\ln 2 \pi)^{2}+\frac{\pi^{2}}{4}\right) \sum_{p=1}^{k} p \chi_{k}(p)\right\} .
\end{aligned}
$$


The results (3.23) and (3.24) contain second derivatives of the generalised zeta function, and there are no formulae analogous to (3.3) available to express these second derivatives in terms of more familiar functions. However, the computer algebra system Maple has the function Zeta (n, s, a) which, for numerical values of $n, s$ and $a$, returns the numerical floating-point value (to any specified precision) of the $n$th derivative of $\zeta(s, a)$. Thus, such derivatives can be considered as known transcendental functions and (3.23) and (3.24) as closed forms. As an example consider the case $k=4$; we then have the summation

$$
\begin{aligned}
\sum_{n=1}^{\infty}(-1)^{n} \frac{(\ln (2 n+1))^{2}}{2 n+1}= & \frac{\pi}{4}\left\{2\left(\zeta^{\prime \prime}(0,1 / 4)-\zeta^{\prime \prime}(0,3 / 4)\right)\right. \\
& \left.-4(\gamma+\ln (2 \pi)) \ln \left(\frac{\Gamma(1 / 4)}{\Gamma(3 / 4)}\right)+(\gamma+\ln (2 \pi))^{2}+\frac{\pi^{2}}{12}\right\} \\
= & -0.15414172442933588340
\end{aligned}
$$

and the integral

$$
\begin{aligned}
\int_{0}^{\infty} \frac{(\ln u)^{2}}{\cosh u} d u= & \frac{\pi}{2}\left\{2\left(\zeta^{\prime \prime}(0,1 / 4)-\zeta^{\prime \prime}(0,3 / 4)\right)\right. \\
& \left.-4 \ln (2 \pi) \ln \left(\frac{\Gamma(1 / 4)}{\Gamma(3 / 4)}\right)+(\ln 2 \pi)^{2}+\frac{1}{4} \pi^{2}\right\} \\
= & 2.35354495271138352907
\end{aligned}
$$

both of which are new.

It is clear that the series obtained by successive differentiations with respect to $s$ followed by setting $s=1$ can be summed using our methods. However, the results become increasingly complicated, involving differences of higher derivatives of $\zeta(s, a)$, and these higher-order derivatives can become numerically large for the values of $a$ involved. Thus exact results for series with high powers of the logarithm are probably of dubious numerical value. We now demonstrate how such series, when of the alternating type, can be summed using Boole's summation formula [9]. Boole's formula, which is not as well known as it deserves to be, states that

$$
\sum_{n=N}^{\infty}(-1)^{n} g(n+h)=\frac{(-1)^{N}}{2} \sum_{p=0}^{m-1} \frac{E_{p}(h)}{p !} g^{(p)}(N)+R_{m},
$$

where $E_{p}(h), p=1,2, \ldots$, are the Euler polynomials and $R_{m}$ is a remainder. The sum on the right-hand side of (3.27) may be either convergent as $m \rightarrow \infty$ or asymptotic, and the validity of Boole's formula requires that $g(x)$ and its derivatives tend to zero as $x \rightarrow \infty$.

As a specific example, suppose that $g(x)=(\ln (2 x+1))^{10} /(2 x+1)$; the series to be summed is first written as

$$
\sum_{n=1}^{\infty}(-1)^{n} \frac{(\ln (2 n+1))^{10}}{2 n+1}=\sum_{n=1}^{N-1}(-1)^{n} \frac{(\ln (2 n+1))^{10}}{2 n+1}+\sum_{n=N}^{\infty}(-1)^{n} \frac{(\ln (2 n+1))^{10}}{2 n+1}
$$

for a suitably chosen $N$. The first series is summed numerically, and Boole's result with $h=0$ and a selected value of $m$ is applied to the second series in (3.28). This process can be carried out using a simple Maple program of less than 10 lines for a 
range of $N, m$ and arithmetic precisions, and the sum calculated to high accuracy. Thus, for example, taking $N=40$ and $m=20$, the sum is found as

$$
\sum_{n=1}^{\infty}(-1)^{n} \frac{(\ln (2 n+1))^{10}}{2 n+1}=-0.53506714857130941959,
$$

correct to 20 decimal places. As with all the series considered in this paper, (3.29) is very slowly convergent; it may be of some interest to point out that the function $g(x)$ attains its maximum value of approximately 454000 when $x \approx 11013$, and thereafter $g(x)$ is monotonically decreasing to zero. This example should convince even the most extreme enthusiast for digital computation that analysis is still indispensable!

The same Maple program used for numerical computation will work of course when applied to the second series in (3.28) for general $N$. Thus, with $t=\ln (2 N)$, we find that

$$
\begin{aligned}
\sum_{n=N}^{\infty}(-1)^{n} & \frac{(\ln (2 n+1))^{10}}{2 n+1}=\frac{(-1)^{N}}{4 N}\left\{t^{10}-\frac{t^{8}\left(t^{2}-15 t+45\right)}{4 N^{2}}\right. \\
& +\frac{5 t^{6}\left(12 t^{4}-250 t^{3}+1575 t^{2}-3600 t+2520\right)}{192 N^{4}} \\
& -\frac{61 t^{4}\left(2 t^{6}-49 t^{5}+406 t^{4}-1470 t^{3}+2450 t^{2}-1764 t+420\right)}{128 N^{6}} \\
& \left.+O\left(t^{10} / N^{8}\right)\right\} .
\end{aligned}
$$

Taking $N=1000$ in (3.28) and using (3.30) to estimate the infinite series produces, to 20 decimal places, the sum -0.53506714857130941962 , which agrees with (3.29) to 19 decimal places.

An alternative derivation of (3.29) proceeds by taking $h=1 / 2$ and $g(x)=$ $(\ln (2 x))^{10} /(2 x)$ in (3.27). Further, it is of interest to note that the Maple command

$\operatorname{evalf}\left(\operatorname{Sum}\left((-1)^{\wedge} n * \ln (2 * n+1) \wedge 10 /(2 * n+1), n=1 \ldots\right.\right.$ infinity $\left.), 20\right) ;$

also delivers (3.29); the method used appears to be a variant of the nonlinear Shanks transformation. Boole's result can be used to sum series of the more general form

$$
\sum_{n=0}^{\infty} \frac{(-1)^{n}(\ln (2 n+1))^{\nu}}{(2 n+1)^{s}}
$$

for arbitrary $\nu$ and for $s>0$; all the numerical values of alternating sums in this paper have also been checked using it. For nonalternating series the EulerMaclaurin summation formula is used instead of Boole's result, a technique which is very well known.

\section{Sums AND INTEGRALS WHEN $\chi_{k}(k-1)=1$}

We now turn to the case $\chi_{k}(k-1)=1$ and state

Theorem 4.1. Let $\chi_{k}(n)$ be a nonprincipal character with $\chi_{k}(k-1)=1$. Then

$$
\sum_{n=2}^{\infty} \frac{\chi_{k}(n) \ln n}{n}=\frac{1}{k^{\frac{1}{2}}}\left\{-2(\gamma+\ln (2 \pi)) \ln \prod_{p=1}^{k} \Gamma(p / k)^{\chi_{k}(p)}+\sum_{p=0}^{k} \chi_{k}(p) \zeta^{\prime \prime}(0, p / k)\right\}
$$


and

$$
\begin{aligned}
\int_{0}^{\infty} \frac{\ln u}{1-e^{-k u}}\left(\sum_{n=1}^{k} \chi_{k}(n) e^{-n u}\right) d u & \\
& =\frac{1}{k^{\frac{1}{2}}}\left\{2 \ln (2 \pi) \ln \prod_{p=1}^{k} \Gamma(p / k)^{\chi_{k}(p)}-\sum_{p=1}^{k} \chi_{k}(p) \zeta^{\prime \prime}(0, p / k)\right\}
\end{aligned}
$$

Proof. To establish the summation formula it is first noted that the derivation of (3.4) holds when $-k$ is replaced by $k$ in the $L$-functions. However, setting $s=0$ in (2.4) shows that $L_{k}(0)=0$, and hence

$$
\sum_{p=1}^{k} p \chi_{k}(p)=0
$$

when $\chi_{k}(k-1)=1$. Again, from (2.8) it follows that

$$
L_{k}^{\prime}(0)=\sum_{p=1}^{k} \chi_{k}(p) \ln \Gamma(p / k)
$$

where (4.1) has been used. Similarly, a double differentiation of (2.8) demonstrates that

$$
L_{k}^{\prime \prime}(0)=\sum_{p=1}^{k} \chi_{k}(p) \zeta^{\prime \prime}(0, p / k)-2 \ln k \sum_{p=1}^{k} \chi_{k}(p) \ln \Gamma(p / k) .
$$

To evaluate $L_{k}^{\prime}(1)$, and hence the required sum, we differentiate the functional relation (2.4) with respect to $s$ and let $s \rightarrow 1$. This shows that

$$
L_{k}^{\prime}(1)=\frac{1}{k^{\frac{1}{2}}}\left\{2(\gamma+\ln (2 \pi / k)) L_{k}^{\prime}(0)-L_{k}^{\prime \prime}(0)\right\},
$$

and by combining (3.1) (with $s=1$ ), (4.2), (4.3) and (4.4) we obtain the required summation: when $\chi_{k}(k-1)=1$,

$$
\sum_{n=2}^{\infty} \frac{\chi_{k}(n) \ln n}{n}=\frac{1}{k^{\frac{1}{2}}}\left\{-2(\gamma+\ln (2 \pi)) \ln \prod_{p=1}^{k} \Gamma(p / k)^{\chi_{k}(p)}+\sum_{p=0}^{k} \chi_{k}(p) \zeta^{\prime \prime}(0, p / k)\right\} .
$$

To evaluate the integral in Theorem 4.1 it is first noted that (3.9) still remains valid with $-k$ replaced by $k$ on the left-hand side. Now $L_{k}^{\prime}(1)$ is given by the negative of the right-hand side of (4.5), and from (2.4)

$$
\begin{aligned}
L_{k}(1) & =\frac{2}{k^{\frac{1}{2}}} \lim _{s \rightarrow 1} L_{k}(1-s) \Gamma(1-s) \\
& =\frac{2}{k^{\frac{1}{2}}} L_{k}^{\prime}(0)=\frac{2}{k^{\frac{1}{2}}} \sum_{p=1}^{k} \chi_{k}(p) \ln \Gamma(p / k)
\end{aligned}
$$

from (4.2). Using this information in (3.9), the definite integral of Theorem 4.1 follows immediately. Clearly, a generalisation of this integral analogous to (3.11) can be constructed. 
We now give two examples of Theorem 4.1 .

(a) $k=5$. There is a nonprincipal character with $k=5$ whose nonzero values are $\chi_{5}(1)=1, \chi_{5}(2)=-1, \chi_{5}(3)=-1$ and $\chi_{5}(4)=1$. Theorem 4.1 provides the nonalternating sum

$$
\begin{aligned}
\sum_{n=0}^{\infty}\left\{\frac{\ln (5 n+1)}{5 n+1}\right. & \left.-\frac{\ln (5 n+2)}{5 n+2}-\frac{\ln (5 n+3)}{5 n+3}+\frac{\ln (5 n+4)}{5 n+4}\right\} \\
= & \frac{1}{\sqrt{5}}\left\{\zeta^{\prime \prime}(0,1 / 5)-\zeta^{\prime \prime}(0,2 / 5)-\zeta^{\prime \prime}(0,3 / 5)+\zeta^{\prime \prime}(0,4 / 5)\right. \\
& -2(\gamma+\ln (2 \pi)) \ln ((1+\sqrt{5}) / 2)\} \\
= & -0.35624046703076149886
\end{aligned}
$$

and the integral result can be expressed as

$$
\begin{aligned}
\int_{0}^{\infty} \ln u & \frac{\sinh u}{2 \cosh 2 u+2 \cosh u+1} d u \\
& =\frac{1}{2 \sqrt{5}}\left\{-\zeta^{\prime \prime}(0,1 / 5)+\zeta^{\prime \prime}(0,2 / 5)+\zeta^{\prime \prime}(0,3 / 5)-\zeta^{\prime \prime}(0,4 / 5)\right. \\
& =0.05390093199632965184 .
\end{aligned}
$$

Note that in (4.7) and (4.8), quotients of products of gamma functions are simplified using the relation $\Gamma(z) \Gamma(1-z)=\pi / \sin (\pi z)$.

(b) $k=8$. For $k=8$ there is a second nonprincipal character with nonvanishing values $\chi_{8}(1)=1, \chi_{8}(3)=-1, \chi_{8}(5)=-1$ and $\chi_{8}(7)=1$. The sum and integral given by Theorem 4.1 are

$$
\begin{aligned}
\sum_{n=0}^{\infty}(-1)^{n} & \left\{\frac{\ln (4 n+1)}{4 n+1}-\frac{\ln (4 n+3)}{4 n+3}\right\} \\
= & \frac{1}{2 \sqrt{2}}\left\{\zeta^{\prime \prime}(0,1 / 8)-\zeta^{\prime \prime}(0,3 / 8)-\zeta^{\prime \prime}(0,5 / 8)+\zeta^{\prime \prime}(0,7 / 8)\right. \\
& =-0.39395000150641812877
\end{aligned}
$$

and

$$
\begin{aligned}
\int_{0}^{\infty} \ln u & \frac{\sinh u}{\cosh 2 u} d u \\
= & \frac{1}{2 \sqrt{2}}\left\{-\zeta^{\prime \prime}(0,1 / 8)+\zeta^{\prime \prime}(0,3 / 8)+\zeta^{\prime \prime}(0,5 / 8)-\zeta^{\prime \prime}(0,7 / 8)\right. \\
& =0.03421463013545748632 .
\end{aligned}
$$

It is interesting to compare (4.9) and (4.10) with (3.16) and (3.17); clearly further results can be generated by the addition and subtraction of these sums and integrals. 


\section{Concluding REMARKS}

In this paper we have given closed-form evaluations of a class of sums and integrals derived essentially from the differentiation of Dirichlet $L$-functions. Many of the results are new, and various generalisations have been indicated. All the series are slowly convergent, and their direct summation poses some difficulty.

The numerical evaluation of series of the form (3.1) for $s \neq 1$ is immediate from (3.2); it is therefore a reasonable suggestion that results such as (3.8) might be found directly by letting $s \rightarrow 1$ in the right-hand side of (3.2). Indeed, using the result [8] that

$$
\lim _{s \rightarrow 1}\left\{\zeta(s, a)-\frac{1}{s-1}\right\}=-\frac{\Gamma^{\prime}(a)}{\Gamma(a)},
$$

and (2.1), it follows immediately that

$$
\lim _{s \rightarrow 1} \sum_{p=1}^{k} \chi_{k}(p) \zeta(s, p / k)=-\sum_{p=1}^{k} \chi_{k}(p) \frac{\Gamma^{\prime}(p / k)}{\Gamma(p / k)} .
$$

To evaluate the limit as $s \rightarrow 1$ of the second sum of derivatives in (3.2) it is necessary to use 2.7). Forming the required derivatives and taking the limit leads to a combination of seemingly intractable integrals of the form

$$
\int_{0}^{\infty}\left(-y \ln \left(a^{2}+y^{2}\right)+2 a \arctan (y / a)\right) \frac{d y}{\left(a^{2}+y^{2}\right)\left(e^{2 \pi y}-1\right)}
$$

for various values of $a$. Thus, the approach of section 2 is to be preferred.

I am grateful to an anonymous referee and to Professor Nico Temme for helpful observations and suggestions which have improved the presentation of this paper.

\section{REFERENCES}

[1] D. Borwein, J. M. Borwein, R. Shail and I. J. Zucker, Energy of static electron lattices, J. Phys A: Math. Gen., 21 (1988), 1519-1531. MR 89f:82070

[2] R. Shail, Some logarithmic lattice sums, J. Phys A: Math. Gen., 28 (1995), 6999-7009. MR 97a:11129

[3] I. S. Gradshteyn and I. M. Ryzhik, Table of Integrals, Series and Products, Academic Press, 1980. MR 81g:30001

[4] K. Chandrasekharan, Introduction to Analytic Number Theory, Springer-Verlag, 1968. MR 40:2593

[5] E. Landau, Handbuch der Lehre von der Verteilung der Primzahlen, Teubner, 1909. MR 16:904d (reprint)

[6] I. J. Zucker and M. M. Robertson, Some properties of Dirichlet L-series, J. Phys A: Math. Gen., 9 (1976), 1207-1214. MR 54:253

[7] I. J. Zucker and M. M. Robertson, Exact values for some two-dimensional lattice sums, J. Phys A: Math. Gen., 8 (1975), 874-881. MR 54:9515

[8] E. T. Whittaker and G. N. Watson, A Course of Modern Analysis, Cambridge University Press, 1952. MR 31:2375 (reprint)

[9] N. M. Temme, Special Functions, Wiley Interscience, 1996. MR 97e:33002

Department of Mathematics and Statistics, University of Surrey, Guildford, Surrey GU2 5XH, UK

E-mail address: r.shail@surrey.ac.uk 